Artículo original

\title{
FORMULACIÓN E IMPLEMENTACIÓN DE UN SOFTWARE SOBRE SISTEMAS DE ESCALERAS DE CONCRETO ARMADO EN PROYECTOS DE INGENIERIA CIVIL
}

FORMULATION AND IMPLEMENTATION OF A SOFTWARE ON ARMED CONCRETE STAIRS SYSTEMS IN CIVIL ENGINEERING PROJECTS

Ing. Jorge Enrique Alvarez Ruffrán Mtro. Edgar Hipólito Chaparro Quispe

Mtro. Dina Marlene Cotrado Flores

Información del artículo:

Recibido: 18/08/2018.

Aceptado: 17/11/2019

${ }^{1}$ Ingeniero Civil

2.Maestro, docente de la Facultad de Ingeniería en la Universidad Privada de Tacna 
Julio - Diciembre del 2019. 


\section{Resumen}

La poca regulación existente en nuestro país referida a la construcción hace resaltable el hecho de la existencia de una gran deficiencia en el diseño como en la ejecución de los mismos. Uno de los componentes afectados y el tratado en este artículo son los sistemas de escaleras de concreto armado, sistemas poco valorados, pero de vital importancia en una edificación, los cuales presentan una falencia en su diseño por diversos factores, para lo cual se planteó realizar un software que analice y diseñe diversos sistemas de escaleras de concreto armado a fin de llegar a implementarlo en diversos proyectos, lográndose la realización del mismo y concluyendo un impacto favorable en la Ingeniería Civil.

Palabras Clave: Análisis, Concreto Armado, Diseño, Escaleras, Software.

\section{Abstract}

The little regulation existing in our country regarding construction highlights the fact of the existence of a great deficiency in the design as in the execution of the same. One of the affected components and the one dealt with in this article are the reinforced concrete stairway systems, systems that are not very valued but are of vital importance in a building, which present a design flaw due to various factors, for which purpose a software that analyzes and designs various systems of reinforced concrete stairs in order to implement it in various projects. Achieving the realization of it and concluding a favorable impact on Civil Engineering.

Keywords: Analysis, Design, Software, Stairs, Reinforced Concrete

\section{INTRODUCCIÓN}

Los sistemas de escaleras de concreto armado aunque poco valorados son de vital importancia en una edificación por brindar rigidez a la misma y permitir la circulación de las personas, esta premisa está en total contrariedad con la realidad presentándose falencias en su diseño y construcción, esto debido a varios factores como la poca importancia que le dan los diseñadores y la poca bibliografía actualizada que se tiene referente a este tema, lo último vendría a ser un factor de gran relevancia ya que debido al mismo los diseñadores optan por la utilización de programas que no analizan a profundad y en muchos casos de manera correcta diversos sistemas de escaleras de concreto armado.

Las investigaciones realizadas a los sistemas de escaleras de C.A. nacen desde los años 90 en donde Bangash, M. Y. H., \& Bangash, T. realizo un estudio a profundidad bajo diversas metodologías de análisis, dicho estudio es uno de los más importantes realizados sobre esta estructura, subsiguientes al mismo Fernández Chea en su tesis "Análisis y Diseño de Escaleras", la cual fue adaptada a un libro, realiza un estudio en el cual se detalla diversos sistemas correspondientes a esta estructura, seguido a estos dos autores no se tienen estudios directos relacionados al tema, existen investigadores relacionados indirectamente, realizando un 
estudio de sistemas de Concreto Armado de manera general, como es el caso de Roberto Morales, el cual en su estudio solo toma el caso de escalera Longitudinal y Transversal. Bajo la premisa de la poca bibliografía actualizada que existe sobre esta estructura, se planteó dar una solución de manera directa a la problemática, la cual es realizar un software el cual ayude en diversos proyectos de Ingeniería Civil analizando y diseñando dichos sistemas. Junto a la elaboración del software se deja una fuente de bibliografía confiable respecto al diseño de cada tipo de sistema tratado en el software de forma que el profesional pueda llegar a comprender la forma de análisis y diseño del mismo, de manera que no solo sea un aplicativo que se use de forma sistemática sin entender los detalles del procesamiento. Interpretando el impacto y la repercusión que tiene este tipo de proyectos en la población, se deja una base para la programación, la cual puede ser usada para cualquier rama de la ingeniería. A manera de motivar al lector a ampliar el horizonte a la tecnología que se tiene en la actualidad, pudiendo cualquier persona con conocimientos y pautas básicas realizadas en este estudio realizar un programa aplicativo para dar solución a una problemática.

\section{METODOLOGÍA}

Para el análisis y diseño de los diversos sistemas de escaleras de C.A. se exploró bases establecidas por diversos autores como lo son Bangash, M. Y. H., \& Bangash y Fernandez Chea, estableciendo diversas formas de análisis en casos particulares de escaleras. Como se muestra en la Tabla 1, se eligió la más conveniente al acercarse a un caso realista y que conlleve accesibilidad en la programación. Se cuenta con una base de diseño para cada caso y seguida a esta con un algoritmo base para proceder a la programación del mismo. Se respetó la Normativa Peruana E.060 (Concreto Armado), para el diseño de los diversos sistemas.

Tabla 1. Metodología de Análisis en algunos Sistemas de Escaleras de C.A.

\begin{tabular}{|c|c|c|}
\hline Tipo de Escalera & Métodos Análisis & Método Elegido \\
\hline Helicoidal & $\begin{array}{ll}\text { - } & \text { M. de Bergman } \\
\text { - } & \text { M. de Nicloski } \\
\text { - } & \text { M. de Mattock } \\
\text { - } & \text { M. de Morgan } \\
\text { - } & \text { M. de Scordelis } \\
\text { - } & \text { M. de Arya y Praskash } \\
\text { - } & \text { M. de Menn } \\
\end{array}$ & M. de Bergman \\
\hline Ortopoligonal & $\begin{array}{l}\text { - } \quad \text { M. de la Analogía de la Columna } \\
\text { - } \quad \text { M. Viga con Carga Repartida } \\
\text { - } \quad \text { M. Viga con Carga Puntual } \\
\end{array}$ & $\begin{array}{l}\text { M. Viga con Carga } \\
\text { repartida }\end{array}$ \\
\hline Apoyada Transv. & $\begin{array}{ll}\text { - } & \text { A. como viga triangular } \\
\text { - } & \text { A. como viga rectangular }\end{array}$ & $\begin{array}{l}\text { A. como viga } \\
\text { triangular }\end{array}$ \\
\hline
\end{tabular}


En el entorno de la programación se cuenta con una gran variedad de interfaces de programación como lenguajes, pudiendo llegar al mismo programa final bajo diversas herramientas. Para el software realizado se optó por usar el lenguaje de programación propio del programa MatLab (Matrix Laboratory), ya que esta es una herramienta orientada a la ingeniería y que brinda grandes posibilidades de programación. A la par del mismo es una herramienta fácil al entendimiento. Se obtuvo acceso a este por medio de una licencia estudiantil con la cual el autor conto al estar llevar estudios en la Universidad Privada de Tacna. Respecto al código base del programa, las diversas fases de elaboración del software (véase la figura 1) y las herramientas usadas para la elaboración del mismo se deja toda la información necesaria en la Universidad Privada de Tacna, lugar donde se dio forma a este proyecto, esperándose en un futuro que profesionales que deseen orientar su investigación en este camino cuenten con una base de información. Una de los objetivos de un software es poder cumplir los estándares de calidad de un programa competitivo en el mercado actual, por lo cual una vez terminada la elaboración del programa se realizaron formularios de calidad del software a diversos profesionales de la rama, con el fin de obtener un análisis numérico del cumplimiento de los objetivos, analizar el impacto que tiene en el medio y a manera de solucionar errores en la programación e implementar nuevas herramientas sugeridas por los usuarios.

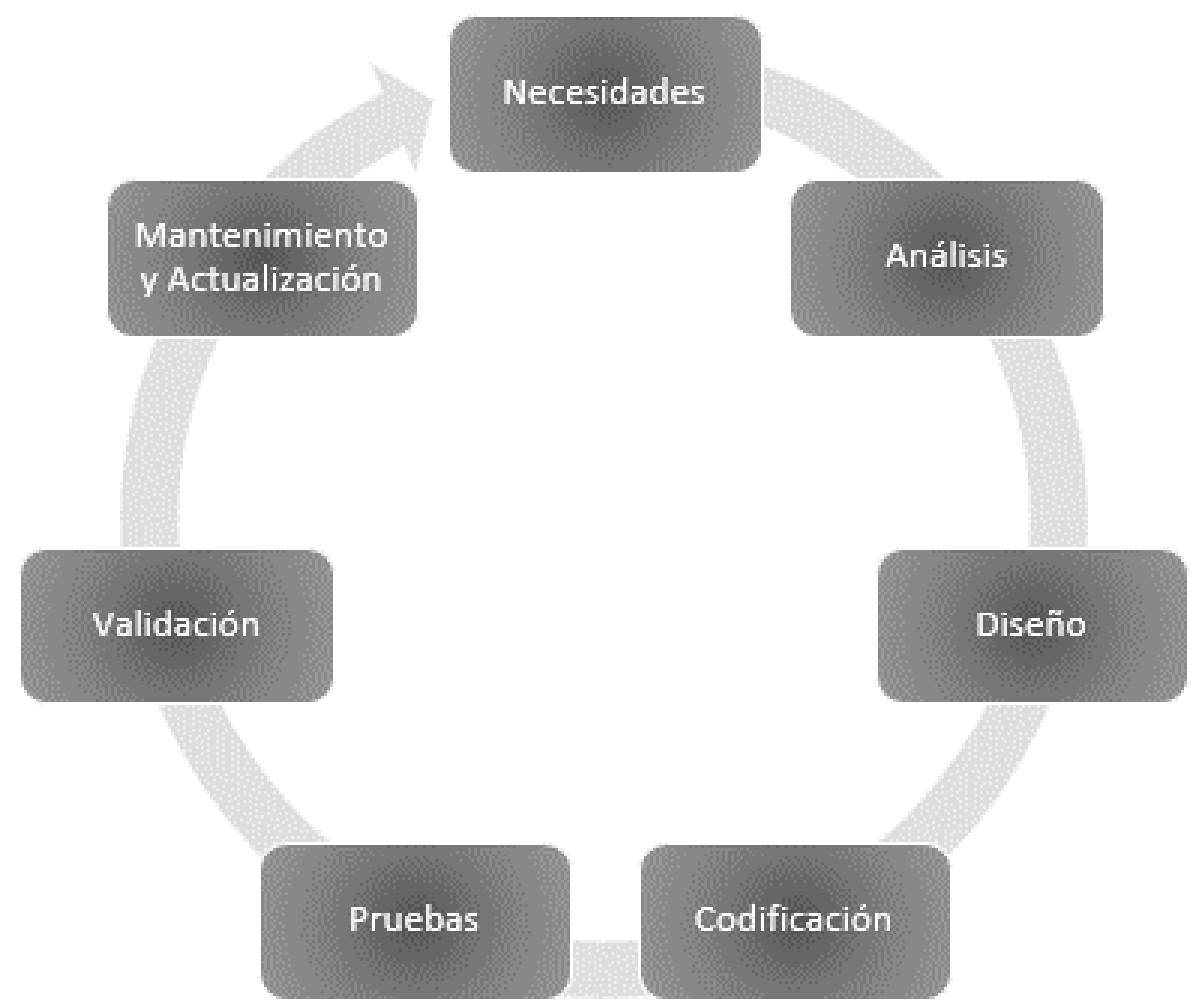

Figura 1. Ciclo de Desarrollo del Software desde el Punto de vista de la Ing. del Software. 
Implementación y Actualización del Software. Al cumplir los objetivos trazados se procede a obtener un software listo para la utilización en diversos proyectos de la ingeniería. Por lo cual, con apoyo de la Universidad Privada de Tacna, en el proceso de elaboración se procede a patentar el software ante Indecopi a manera de obtener los derechos del mismo. Esto a manera de poder lanzarlo a un mercado y que puede ser utilizado en los proyectos de ingeniería, de tal manera que siguiendo al ciclo de desarrollo de un software (véase la figura 1 ), empezar a incorporar nuevas herramientas en un futuro a través de la actualización del mismo.

\section{RESULTADOS}

Como se mencionó anteriormente en la fase de metodología de análisis y diseño, se tienen diversas metodologías de análisis previstas para el mismo sistema de escalera. Esto se da debido a la metodología del propio autor a llevar su planteamiento a un caso más realista para el tipo de escalera que se está evaluando. En la Tabla 2 se muestran un caso de variación en el análisis de la escalera, el cual no llega repercutir de gran manera al diseño propio de la misma.

Tabla 2. Comparativo de Análisis-Diseño presentado entre el software y Roberto Morales Morales en su libro "Diseño en Concreto Armado", para un sistema Longitudinal.

\begin{tabular}{|c|c|c|c|}
\hline Autor & Análisis & Diseño & Cuantía de Acero \\
\hline Software: Staircases & Momento maximo: & Cant. de Acero: & $\varnothing 1 / 2^{\prime \prime} @ 26.4$ \\
& 2.172 tn-m & $4.805 \mathrm{~cm} 2$ & $\mathrm{~cm}$ \\
\hline $\begin{array}{c}\text { Roberto Morales } \\
\text { Morales }\end{array}$ & $\begin{array}{c}\text { Momento maximo: } 2.49 \\
\text { tn-m }\end{array}$ & $\begin{array}{c}\text { Cant. de Acero: } \\
4.49 \mathrm{~cm} 2\end{array}$ & $\varnothing 1 / 2^{\prime \prime} @ 28 \mathrm{~cm}$ \\
\hline
\end{tabular}

El fiel cumplimiento de los objetivos y llegar a tener los estándares de calidad de un programa competitivo en el mercado actual, se ve reflejado con los formularios de calidad del software, datos numéricos que conllevan a entender la repercusión del software en la población, estos datos se ven reflejados en la tabla 3. Y nos muestran que el programa tiene un nivel de aceptación positivo con respecto al entorno profesional al cual está dirigido.

Cumpliéndose los objetivos base se tiene un software que puede lanzarse al mercado y junto a este, la implementación de nuevas herramientas de ayuda a fiel cumplimiento de la fase de mantenimiento y evolución del software, esto es gracias a la propia necesidad del usuario frente al uso de nuevas y mejores herramientas. En la figura 2 y 3 se muestra un claro ejemplo de evolución del software frente a la necesidad del usuario teniendo un antes y después de una de las diversas interfaces graficas del software para un caso particular de sistema de escalera de concreto armado. 
Tabla 3. Resultados obtenidos de los formularios de Calidad del Software

\begin{tabular}{|c|c|}
\hline Aspecto & Resultado Obtenido \\
\hline $\begin{array}{l}\text { Repercusión } \\
\text { en la } \\
\text { Profesión }\end{array}$ & $\begin{array}{l}\checkmark \text { En un } 93 \% \text { de los formularios entregados se encuentran profesionales } \\
\text { satisfechos o muy satisfechos con el software. } \\
\checkmark \text { En un } 80 \% \text { de los formularios entregados se encuentran profesionales que } \\
\text { recomiendan a otros en el uso del software. }\end{array}$ \\
\hline $\begin{array}{l}\text { Fiabilidad del } \\
\text { Software }\end{array}$ & $\begin{array}{l}\checkmark \text { Un } 90 \% \text { de los formularios entregados dieron como resultado que el } \\
\text { software es de confianza con un nivel entre } 4 \text { a } 5 \text {, de una escala de } 1 \text { al } 5 \text {. }\end{array}$ \\
\hline $\begin{array}{l}\text { Presencia de } \\
\text { errores en la } \\
\text { Programación }\end{array}$ & $\begin{array}{l}\checkmark \text { En su totalidad }(100 \%) \text {, durante la prueba del software no se encontraron } \\
\text { bugs o problemas en la programación que afectaran el desarrollo del } \\
\text { mismo. }\end{array}$ \\
\hline $\begin{array}{l}\text { Eficiencia del } \\
\text { Software }\end{array}$ & $\begin{array}{l}\checkmark \text { Un } 70 \% \text { de los formularios entregados dieron como resultado que el } \\
\text { software es de confianza con un nivel entre } 4 \text { a } 5 \text {, de una escala de } 1 \text { al } 5 \text {. }\end{array}$ \\
\hline $\begin{array}{l}\text { Facilidad de } \\
\text { Uso }\end{array}$ & $\begin{array}{l}\checkmark \text { Un } 80 \% \text { de los formularios brindaron un puntaje entre } 3 \text { a } 5 \text { en una escala } \\
\text { del } 1 \text { al } 5 \text { en cuando a la facilidad de instalación. } \\
\checkmark \text { Un } 90 \% \text { de los formularios brindaron una respuesta entre } 4 \text { a } 5 \text { en } \\
\text { facilidad de ingreso de resultados ejecución entre otras características del } \\
\text { software en sí. }\end{array}$ \\
\hline Portabilidad & $\begin{array}{l}\checkmark \text { Un } 95 \% \text { de los formularios de prueba brindaron una calificación de } 4 \text { a } 5 \\
\text { en el nivel de portabilidad del software. }\end{array}$ \\
\hline
\end{tabular}

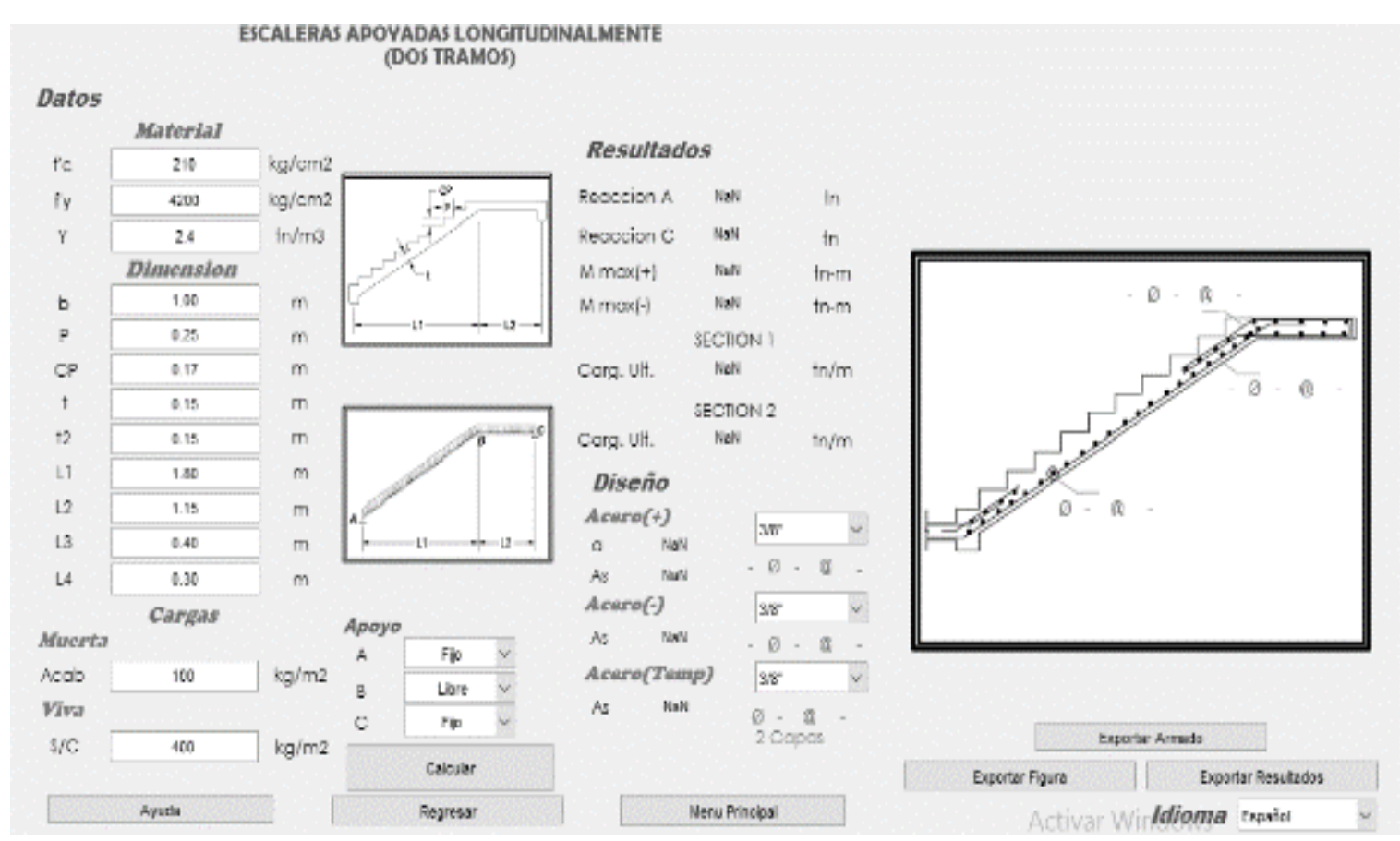

Figura 2. Interfaz Gráfica del Software "StairCases", para un caso de escalera en las primeras etapas de desarrollo. 


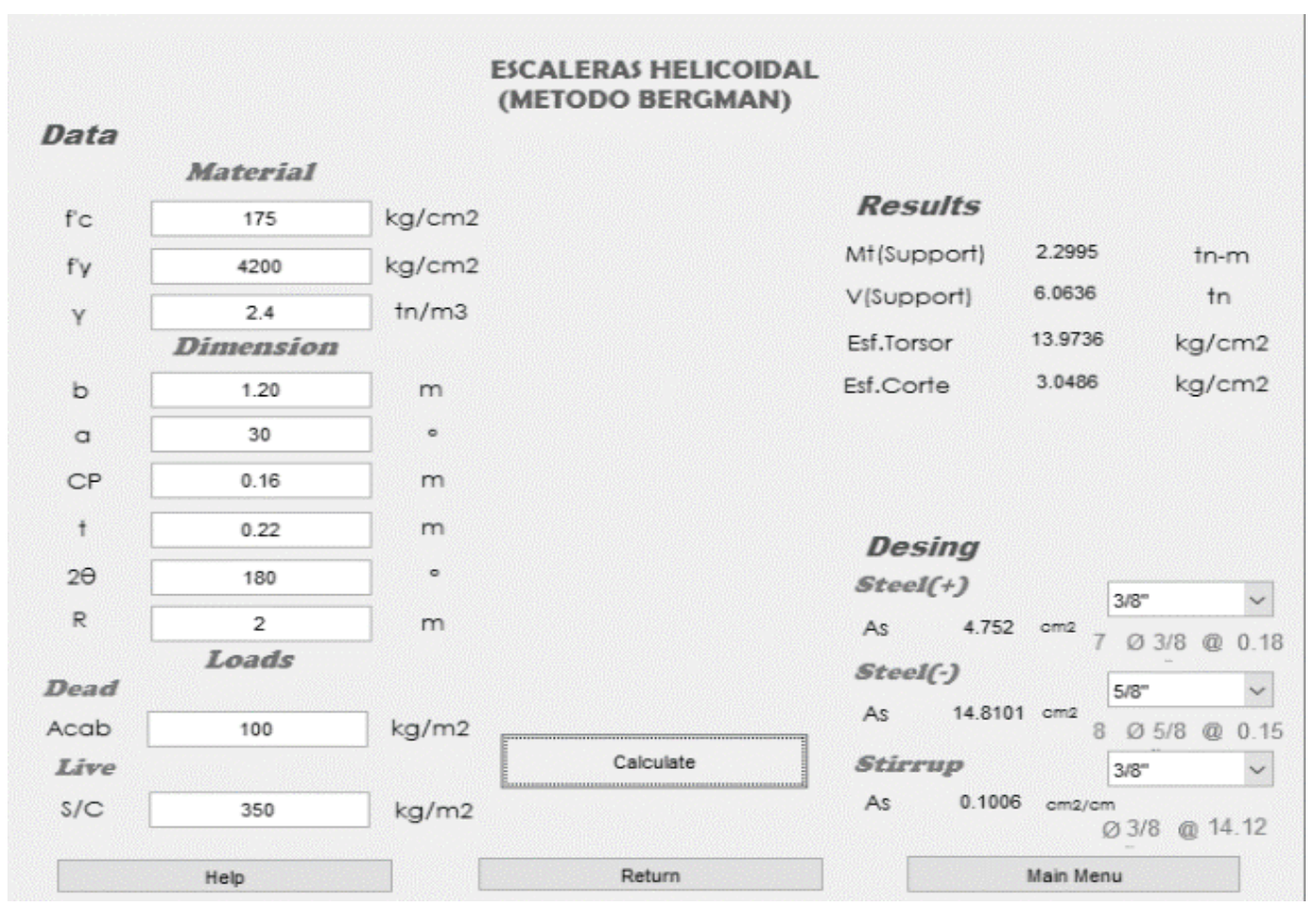

Figura 3. Interfaz Gráfica del Software "StairCases", para un caso de escalera en las últimas etapas de desarrollo.

Fases para la elaboración de un diseño estructural en un proyecto. En la elaboración de proyectos en la Ingeniería Civil, con respecto al diseño de estructuras. Se cuentan con diversas etapas por la cual debe pasar el sistema estructural, En la figura 4 se muestra un diagrama de evolución de un diseño en un proyecto de Ingeniería. Debido al fiel cumplimiento de estas fases el software se ve orientado a cumplir cada una de las mismas incorporando nuevas herramientas en su código, en la tabla 4 se muestra la herramienta usada para el cumplimiento de las diversas fases.

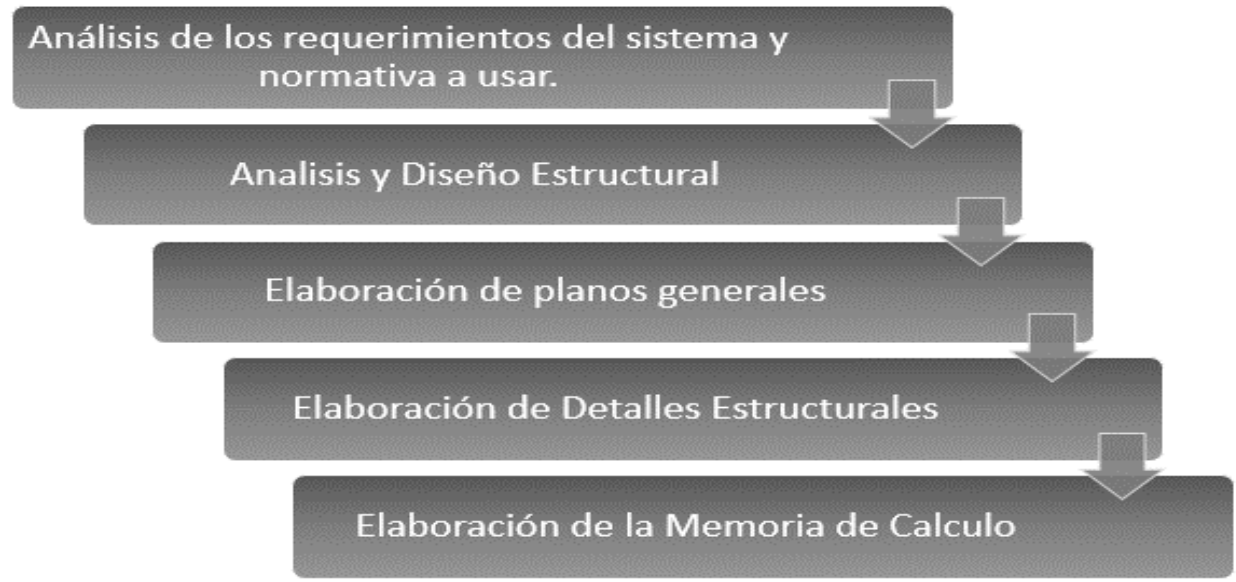

Figura 4. Diagrama de evolución de un diseño estructural, frente a un proyecto de Ingeniería. 
Tabla 4. Herramientas implementadas para cada fase de desarrollo del diseño estructural.

\begin{tabular}{|c|c|}
\hline $\begin{array}{c}\text { Fase de Desarrollo } \\
\begin{array}{c}\text { Requerimientos del } \\
\text { sistema y normativa a } \\
\text { usar }\end{array}\end{array}$ & $\begin{array}{l}\text { Interfaz de Ayuda. La cual presenta los diversos tipos de } \\
\text { carga correspondiente con el tipo de uso y diversas } \\
\text { solicitaciones de diseño. }\end{array}$ \\
\hline $\begin{array}{c}\text { Análisis y diseño } \\
\text { Estructural }\end{array}$ & $\begin{array}{l}\text { Interfaz Gráfica General. Varían notoriamente } \\
\text { dependiendo del tipo de Escalera, estas conllevan } \\
\text { principalmente ingreso de datos, variaciones en los } \\
\text { resultados y análisis/diseño del sistema. }\end{array}$ \\
\hline $\begin{array}{c}\text { Elaboración de planos } \\
\text { Generales }\end{array}$ & $\begin{array}{l}\text { Exportación Armado. Herramienta de exportación del } \\
\text { plano de Diseño General a Autocad bajo formato dxf. Esta } \\
\text { herramienta se ha implementado gracias al uso de la } \\
\text { herramienta DXFLib obtenida de la web MathWorks. }\end{array}$ \\
\hline $\begin{array}{c}\text { Elaboración de Detalles } \\
\text { Estructurales }\end{array}$ & $\begin{array}{l}\text { Exportar figura. Herramienta implementada por el propio } \\
\text { Mathworks e introducida como opción en el programa, la } \\
\text { cual da comandos de modificación en la figura como } \\
\text { exportación de la misma en diversos formatos. }\end{array}$ \\
\hline $\begin{array}{c}\text { Elaboración de la } \\
\text { Memoria de Calculo }\end{array}$ & $\begin{array}{l}\text { Exportar Resultados. Herramienta implementada la cual } \\
\text { exporta a Word los resultados obtenidos junto con los } \\
\text { datos del sistema Estructural. }\end{array}$ \\
\hline
\end{tabular}

\section{DISCUSIÓN}

Se puede observar discrepancia entre los resultados obtenidos por el software, respecto al análisis y diseño, y los resultados obtenidos por algunos autores, frente al mismo sistema estructural. Un claro ejemplo se ve visualizado en la Tabla 2, en este particular caso la variación de resultado es debido al planteamiento del autor con respecto al metrado de cargas de la escalera, otras variaciones de resultados pueden ser debido al cálculo de momentos, entre otros. Los resultados brindados en el Libro StairCases por M.Y H. Bangash \& T.Bangash guardan relación con los resultados obtenidos en la presente investigación, pero se tiene que resaltar que en algunos casos se eligió un método diferente con una igual precisión de resultados, esto es debido a obtener una mejor facilidad en la programación y un menor tiempo de procesamiento de datos. La aceptación de una de las premisas con la cual se realizó la investigación, la cual define como el software un método para reducir un cálculo extenso sin dejar de ser preciso y eficaz, es positiva, resultado obtenido del nivel de aceptación que tuvieron los formularios de calidad del software al sector que está dirigido el mismo. A través del trabajo realizado y los resultados que brindaron el estudio estadístico se concluye que un software base que se oriente a una problemática bien definida, puede causar un gran impacto 
al público al cual se desea acceder. El poco conocimiento y la poca importancia de los profesionales frente a un tema en específico, como son los sistemas de escaleras de C.A. en este caso, causa poca elaboración de estudios e investigaciones con respecto al propio tema, esto debido a la idea de que los sistemas de "poca importancia", no serán de gran impacto en una edificación en caso de falencias en su análisis, diseño y posterior construcción. El principal motivo de la poca realización de proyectos correspondientes a la elaboración de software que conlleven una programación se basa en el paradigma del profesional, el cual está basado en que la programación es una tarea que conlleva un gran estudio y grandes esfuerzos para el entendimiento del mismo, idea totalmente errada con respecto a la actualidad, pudiendo un profesional con solo conocimientos básicos de cómo funciona un programa y pautas marcadas en esta investigación, realizar programas básicos solucionando cualquier problemática que se tenga respecto a su carrera.

\section{REFERENCIAS BIBLIOGRÁFICAS:}

Bangash, M. \& Bangash, T. (1999). Staircases Structural analysis and Design. Canadá: A.A.Balkema.

Fernandez C. C. (2001). Análisis y Diseño de Escaleras. Lima, Perú: Ciencias.

Ortega, G.J. (2015). Diseño de estructuras de Concreto Armado -Tomo Il. Lima, Perú: Macro.

Morales, M.R. (2016). Diseño en concreto Armado. Lima, Perú: Instituto de la Construcción y Gerencia.

R.N.E. E060. Concreto Armado. (2012).

Kwiatek, G. (Noviembre, 2017). DXFLib

[DXFLib is a library written in MATLAB that allows to create simple AutoCAD DXF files] Recuperado de https://la.mathworks.com/matlabcentral/fileexchange/33884-dxflib Patente

StairCases (2018). Patente $N^{\circ}$.Part.Registral: 00104-2018. Peru: INDECOPI. Dirección de Derecho de Autor 